\title{
Association of maternal whole blood fatty acid status during the prenatal period with term birth dimensions: a cross-sectional study \\ by Husna9 Yetti
}

Submission date: 20-Dec-2019 03:54PM (UTC+0800)

Submission ID: 1237377324

File name: dr.Husna-Jurnal9.pdf (599.42K)

Word count: 7929

Character count: 37432 
Xiaofang Jia, Masahiro Tagawa, Hiroshi Yatsuya, Hisao Naito, Yumi Hayashi, Husna Yetti, Seiko Sasaki, Atsuko Araki, Chihiro Miyashita, Tamiko Ikeno, Reiko Kishi and Tamie Nakajima*

\section{Association of maternal whole blood fatty acid status during the prenatal period with term birth dimensions: a cross-sectional study}

\section{Abstract}

Objective: To investigate selected fatty acid (FA) profiles in maternal whole blood during normal pregnancy and to evaluate their associations with term birth dimensions.

Methods: We characterized nine major maternal blood FAs representing four FA families during the second and third trimester of pregnancy, and explored their associations with birth weight, length, and chest or head circumferences by multivariate regression models, using data from 318 mother-newborn pairs of the Hokkaido Study.

Results: The absolute and/or relative contents of maternal blood docosahexaenoic acid and arachidonic acid were lowest at 35-41 gestational weeks during pregnancy, as was the essential FA status index. Different from palmitic and stearic acids, palmitoleic and oleic acid contents were higher at 35-41 gestational weeks than those

${ }^{*}$ Corresponding author: Tamie Nakajima, PhD, College of Life and Health Sciences, Chubu University, Kasugai 487-8501, Japan, Tel.: +8156851 9655, Fax: +8156851 5370, E-mail: tnasu23@med.nagoya-u.ac.jp; and Department of Occupational and Environmental Health, Nagoya University Graduate School of Medicine, Nagoya, Japan

Xiaofang Jia: Department of Occupational and Environmental Health, Nagoya University Graduate School of Medicine, Nagoya, Japan; and National Institute for Nutrition and Health (former National Institute for Nutrition and Food Safety), Chinese Center for Disease Control and Prevention, Beijing, China

Masahiro Tagawa, Hisao Naito and Husna Yetti: Department of Occupational and Environmental Health, Nagoya University Graduate School of Medicine, Nagoya, Japan

Hiroshi Yatsuya: Department of Public Health, Fujita Health University School of Medicine, Toyoake, Japan

Yumi Hayashi: Department of Pathophysiological Laboratory Sciences, Nagoya University Graduate School of Medicine, Nagoya, Japan Seiko Sasaki: Department of Public Health Sciences, Hokkaido University Graduate School of Medicine, Sapporo, Japan Atsuko Araki, Chihiro Miyashita, Tamiko Ikeno and Reiko Kishi: Center for Environmental Health and Sciences, Hokkaido University, Sapporo, Japan at 23-31 gestational weeks. Three FA components were identified through principal component analysis, and were used in association analysis. Component 3 , which was positively and significantly loaded by eicosapentaenoic acid (EPA), was associated with chest circumference [ $\beta=0.281,95 \%$ confidence interval (CI): $0.006,0.556]$ at 35-41 gestational weeks $(\mathrm{P}=0.046)$. No significant associations were observed for Component 1 and 2 loaded by FAs except EPA.

Conclusion: Maternal blood EPA content may have an important influence on infant chest circumference.

Keywords: Association study; fatty acid status; pregnancy; term birth outcomes.

DOI 10.1515/jpm-2014-0277

Received August 17, 2014. Accepted October 16, 2014. Previously published online December 6, 2014.

\section{Introduction}

Fatty acids (FAs) are essential for life as major sources of energy and structural components of cell membranes [1]. In the human body, long chain saturated FAs (SFAs) and unsaturated FAs of the n-7 and n-9 series can be synthesized from palmitic acid (16:0) [1]. Essential FAs (EFAs) are vital for human health, but cannot be synthesized by humans. Therefore, they have to be consumed with food. There are two families (n-6 and n-3) of EFAs: the parent EFAs linoleic acid (LA, 18:2n-6) and $\alpha$-linolenic acid (ALA, 18:3n-3), and their long chain polyunsaturated FA (LCPUFA) derivatives, such as arachidonic acid (AA, 20:4n-6), eicosapentaenoic acid (EPA, 20:5n-3), and docosahexaenoic acid (DHA, 22:6n-3), respectively [1, 2].

The maternal FA status, especially of polyunsaturated FAs (PUFAs), during normal pregnancy is under active 
investigation [3-5]. A longitudinal study using repeated blood samples of pregnant women from the tenth week of gestation until delivery indicated that the relative amount of LA in plasma phospholipids did not change during pregnancy, whereas that of AA decreased. The DHA steadily declined after a temporary increase until 18 weeks of gestation. The overall maternal EFA status also progressively decreased during pregnancy [3]. Several studies investigated the changes in total plasma FA profiles during pregnancy. There was a significant decrease in the proportion of n-3 PUFAs in plasma from the first to third trimester in a longitudinal study [6]. Several crosssectional studies also reported changes in the composition of total plasmatic FAs during pregnancy: a significant increase in the proportion of palmitic acid and a significant decrease in AA occurred between the first and second trimesters, which were more marked between the second trimester and at delivery [7].

The human fetus is dependent on adequate placental transport of FAs from the maternal circulation, in addition to many other nutrients, for normal development and growth [2]. As birth dimensions have prognostic potential for later development and health [8], associations between neonatal birth dimensions at term birth and selected FA contents in phospholipids of maternal plasma throughout gestation have been increasingly investigated [9-11]. Lower concentrations of most n-3 PUFAs and higher concentrations of AA early in pregnancy were associated with lower birth weight, after adjustment for confounders [9]. Similarly, sig. nificant positive associations were observed between the proportion of DHA (especially early in pregnancy) and birth weight and head circumference [10], while the proportion of AA at late pregnancy and at delivery was negatively associated with birth weight and birth length [10]. These studies suggested that unlike AA, the proportion of DHA in maternal blood during early pregnancy may be positively associated with fetal growth. As FAs are metabolized in the body by the same enzymes, metabolic interactions are often observed [10]. Associations of birth outcomes with individual FA may be affected by metabolic interactions with other FAs. Taken together, to investigate the influence of maternal blood various FA statuses on infant birth outcomes, under the consideration of metabolic interaction of FAs, is required.

The present study aimed to investigate selected FA profiles in maternal whole blood during normal pregnancy and to evaluate their associations with term birth dimensions using a Japanese cohort of pregnant women.

\section{Methods}

\section{General design of the study}

This study was part of the "Hokkaido Study on Environment and Children's Health", a hospital-based prospective cohort study conducted by the Hokkaido University Graduate School of Medicine [12-14]. Briefly, this cohort study is based on pregnant women who delivered at the Sapporo Toho Hospital in Sapporo, Hokkaido, Japan. From July 2002 to October 2005, we approached pregnant women who were between 23 and 35 weeks of gestation, and had no serious illnesses and medical complications. All potential subjects were native Japanese living in Sapporo and the surrounding industrialized areas. The following were the exclusion criteria for study subjects: women with incomplete information regarding their partner, women who had decided to enroll in the Japanese cord blood bank, and women who had decided to deliver their baby at another hospital. Some of the women we approached did not express interest in our study, and some were unable or unwilling to participate in the study. Ultimately, 514 pregnant women were enrolled in the Sapporo cohort study by providing written informed consent. All the women took antenatal and perinatal examinations at the hospital. Medical records of the hospital were utilized to obtain maternal and infant medical information, including multiple births, infant gender, gestational age, birth weight, birth length, birth chest or head circumference, maternal age, maternal height, maternal weight before pregnancy, parity, and medical history during pregnancy. Participants also completed a self-administered questionnaire survey after the second trimester about potential confounders in relation to the past medical history of the mothers and their partners, demographic characteristics, health status during pregnancy, dietary intake during pregnancy, work history during pregnancy, smoking habits, alcohol intake, caffeine intake, household income, education level, and exposure to chemical compounds in their daily life, as described in detail elsewhere [12-14]. All this information was collected to form the Hokkaido study database.

The present study extracted relevant data of eligible mothers and their infants from the database. Measurements of selected FA contents in maternal whole blood were conducted for 493 maternal blood samples in Nagoya University. Approval for this study was obtained from the Institutional Ethical Board for Epidemiologic Studies of Hokkaido University Graduate School of Medicine and from the Ethics Review Committee of Nagoya University Graduate School of Medicine.

\section{Blood sampling}

A 40-mL blood sample was taken from the maternal peripheral vein during the antenatal hospital examinations following enrollment. If the blood could not be taken during pregnancy because of maternal anemia, it was obtained during a 1-week hospitalization after delivery. Blood was obtained once from each woman. All samples were stored at $-80^{\circ} \mathrm{C}$ until analysis. Consistent with published reports of the "Hokkaido Study on Environment and Children's Health" [13], the blood sampling period was categorized into four groups: 23-31 weeks of gestation, 32-34 weeks of gestation, 35-41 weeks of gestation, and within a week after delivery. 


\section{FA profiles in maternal whole blood}

FA levels in maternal blood were determined by GC-MS as described in detail in our earlier study [15] after extracting lipids according to the method of Folch et al. [16]. Nine FA species targeted for measurement included palmitic and stearic acids of SFAs, palmitoleic and oleic acids of monounsaturated FAs (MUFAs), LA and AA of n-6 PUFAs, and ALA, EPA and DHA of n-3 PUFAs. Under the experimental conditions, the detection limits were $2.4 \mu \mathrm{g} / \mathrm{mL}$ for palmitic acid, 1.3 $\mu \mathrm{g} / \mathrm{mL}$ for stearic acid, $0.69 \mu \mathrm{g} / \mathrm{mL}$ for palmitoleic acid, $3.6 \mu \mathrm{g} / \mathrm{mL}$ for oleic acid, and $2.0 \mu \mathrm{g} / \mathrm{mL}$ for each of the others. The data of FAs were also included in the unpublished results of one of our previous studies.

\section{Inclusion of participants}

Ten women were excluded from the study because of miscarriage, stillbirth, moving away before delivery, or voluntary withdrawal from the study. Forty-three mother-infant pairs were excluded because: the mothers had developed pregnancy-induced hypertension $(n=11)$ or gestational diabetes mellitus $(n=1)$; the mothers had delivered multiple infants $(n=7)$; infants had heart failure $(n=1)$; or infants were born preterm (gestational age $<37$ weeks, $n=23$ ). One hundred and thirty-four mother-infant pairs were further excluded as maternal blood had been collected after delivery. Out of the remaining 327 mothers, we obtained whole blood FA levels from 318 subjects, as a result, leaving 318 pairs for the analysis.

\section{Covariates}

Maternal age, height, pre-pregnancy weight, parity, smoking habit and alcohol intake during pregnancy, annual household income as the socio-economic status, and the blood sampling period (gestational age at blood sampling) were maternal covariates [9, 10, 13, 14]. In addition, gestational age at birth, delivery type, and the sex of newborns were neonatal covariates $[9,10,13,14]$. The blood sampling period was used to control variations in FA concentrations that normally occur during the course of pregnancy [3,9]. Parity was classified into two groups: primiparous and multiparous. Both smoking and alcohol intake statuses during pregnancy were dichotomized (yes/no). Information on annual household income was obtained from a self-reported questionnaire. It was divided into four categories: $\leq 3$ million yen, 3-5 million yen, 5-7 million yen, and $\geq 7$ million yen. The delivery type was dichotomized: vaginal or cesarean section.

\section{Statistical analysis}

First, mean FA levels in maternal blood were calculated according to the blood sampling period in order to simulate maternal blood FA changes during pregnancy by one-way ANOVA, followed by Tukey's test for multiple comparisons. Next, the associations of maternal blood FA levels with neonatal birth dimensions such as birth weight, birth length, and chest or head circumferences were studied by linear regression analyses, including the aforementioned covariates as potential confounding factors.
We refer to the sum of the nine measured FAs as the total FA concentration. The relative proportion of a given FA to total FAs was calculated by dividing its concentration by the total concentration of FAs. The EFA status index was calculated, defined as the ratio of the sum of the $n-3$ and n-6 FAs to the sum of the n-7 and $\mathrm{n}-9$ FAs [3]. Data of variables were presented as the mean \pm SEM. If the distribution of a variable was not normal, a logarithm or square root transformation was performed before analysis. Otherwise, a Kruskal-Wallis test was used if parametric test assumptions were not satisfied.

As the nine FAs were highly correlated with one another (Spearman correlation coefficient range: 0.112-0.919), principal component analysis (PCA) was performed on the correlation matrix of the nine measured FAs. Briefly, PCA is a data reduction technique that forms linear combinations of original variables into groups of correlated variables, each accounting for as much of the remaining variance of all of the FAs as possible, as reported elsewhere $[17,18]$. Varimax rotations were used to obtain an orthogonal solution. The first three components were extracted from the present dataset depending on cumulatively explained total variance $(>80 \%$ ), whereas the rest accounted for a fraction of total variance and were excluded from the remaining analysis. The factor loadings of the nine FAs for the three components were outputted, representing the correlations of each component with its corresponding FA, and were used to calculate factor scores for each participant. Individuals with a higher score were indicated to have an FA pattern described by the component more commonly than those with a lower score. Factor scores for each component were treated as continuous variables with a nearly normal distribution. The associations of the three components and birth outcome measures were examined by simple linear regression analyses. Then, multivariate analyses were performed by consecutively including predefined sets of covariates. First, we included factor scores for other components and covariates for maternal physiological characteristics, and neonatal characteristics (Model 1). The delivery type was also included, but only for head circumference. Subsequently, we further added covariates for maternal lifestyle and socio-economic factors (Model 2). Linear regression analyses were also stratified by gestational age at blood sampling to examine the interaction with gestational age. No variables included in the multivariate models had a problem with multicollinearity according to a variance inflation factor $>10$. All statistical analyses were performed using SPSS 17.0 software (Chicago, IL, USA). Results were statistically significant if $\mathrm{P}<0.05$.

\section{Results}

\section{Characteristics of mothers and neonates}

We included 318 mother-infant pairs in the study (Table 1). The differences in smoking status during pregnancy were significant among three blood sampling groups, and no differences were observed for other characteristics of mothers and neonates, which indicated that women with different blood sampling periods were almost homogenous. 
Table 1 Characteristics of mothers and neonates by blood sampling period $(n=318)$.

\begin{tabular}{|c|c|c|c|c|}
\hline \multirow[t]{2}{*}{ Characteristics } & \multicolumn{3}{|c|}{ Blood sampling period (gestational weeks) } & \multirow[t]{2}{*}{ P-value } \\
\hline & $\begin{array}{r}23-31 \\
(n=135)\end{array}$ & $\begin{array}{l}32-34 \\
(n=81)\end{array}$ & $\begin{array}{r}35-41 \\
(n=102)\end{array}$ & \\
\hline \multicolumn{5}{|l|}{ Maternal characteristics } \\
\hline Age (years) & $30.3 \pm 0.4$ & $29.7 \pm 0.5$ & $29.4 \pm 0.5$ & 0.219 \\
\hline Height $(\mathrm{cm})^{\mathrm{a}}$ & $159.0 \pm 0.4$ & $157.9 \pm 0.6$ & $158.2 \pm 0.5$ & 0.242 \\
\hline Pre-pregnancy body weight $(\mathrm{kg})^{a, b}$ & $53.7 \pm 0.7$ & $53.2 \pm 0.9$ & $51.7 \pm 0.7$ & 0.259 \\
\hline Pre-pregnancy BMI $\left(\mathrm{kg} / \mathrm{m}^{2}\right)^{a, b}$ & $21.2 \pm 0.3$ & $21.3 \pm 0.3$ & $20.7 \pm 0.3$ & 0.245 \\
\hline \multicolumn{5}{|l|}{ Parity (times) ${ }^{\mathrm{b}}$} \\
\hline 0 & $72(53.3)$ & $41(50.6)$ & $52(51.0)$ & 0.925 \\
\hline$\geq 1$ & $63(46.7)$ & $39(48.1)$ & $50(49.0)$ & \\
\hline \multicolumn{5}{|l|}{ Past conception (times) } \\
\hline 0 & $52(38.5)$ & $30(37.0)$ & $37(36.3)$ & 0.944 \\
\hline 1 & $45(33.3)$ & $26(32.1)$ & $31(30.4)$ & \\
\hline$\geq 2$ & $38(28.1)$ & 25 (30.9) & $34(33.3)$ & \\
\hline \multicolumn{5}{|l|}{ Education level (years) } \\
\hline$\leq 12$ & $58(43.0)$ & $35(43.2)$ & $49(48.0)$ & 0.706 \\
\hline$\geq 13$ & $77(57.0)$ & $46(56.8)$ & $53(52.0)$ & \\
\hline \multicolumn{5}{|l|}{ Annual household income (million yen) ${ }^{\mathrm{b}}$} \\
\hline$<3$ & $26(19.3)$ & $20(24.7)$ & $22(21.6)$ & 0.880 \\
\hline $3-5$ & $69(51.1)$ & $37(45.7)$ & $47(46.1)$ & \\
\hline $5-7$ & $26(19.3)$ & $14(17.3)$ & $23(22.5)$ & \\
\hline$\geq 7$ & $13(9.6)$ & $10(12.3)$ & $9(8.8)$ & \\
\hline \multicolumn{5}{|l|}{ History of smoking } \\
\hline Yes & $79(58.5)$ & $49(60.5)$ & $57(55.9)$ & 0.816 \\
\hline No & $56(41.5)$ & $32(39.5)$ & $45(44.1)$ & \\
\hline \multicolumn{5}{|l|}{ Smoking during pregnancy } \\
\hline Yes & $21(15.6)$ & 25 (30.9) & $22(21.6)$ & $0.029^{c}$ \\
\hline No & $114(84.4)$ & $56(69.1)$ & $80(78.4)$ & \\
\hline \multicolumn{5}{|l|}{ Alcohol intake during pregnancy } \\
\hline Yes & $49(36.3)$ & $26(32.1)$ & $31(30.4)$ & 0.611 \\
\hline No & $86(63.7)$ & $55(67.9)$ & $71(69.6)$ & \\
\hline Alcohol intake among drinkers during pregnancy ( $\mathrm{g} /$ day) ${ }^{\mathrm{a}}$ & $3.7 \pm 0.7$ & $8.0 \pm 5.8$ & $7.2 \pm 3.4$ & 0.737 \\
\hline \multicolumn{5}{|l|}{ Type of delivery } \\
\hline Vaginal & $113(83.7)$ & $67(82.7)$ & $93(91.2)$ & 0.170 \\
\hline Cesarean section & $22(16.3)$ & $14(17.3)$ & $9(8.8)$ & \\
\hline \multicolumn{5}{|l|}{ Infant characteristics } \\
\hline \multicolumn{5}{|l|}{ Sex } \\
\hline Male & $68(50.4)$ & $42(51.9)$ & $41(40.2)$ & 0.198 \\
\hline Female & $67(49.6)$ & $39(48.1)$ & $61(59.8)$ & \\
\hline Gestational age (weeks) & $39.2 \pm 0.1$ & $39.4 \pm 0.1$ & $39.5 \pm 0.1$ & 0.227 \\
\hline Birth weight $(\mathrm{g})^{\mathrm{a}}$ & $3097.0 \pm 31.0$ & $3076.8 \pm 37.3$ & $3156.8 \pm 33.2$ & 0.277 \\
\hline Birth length $(\mathrm{cm})^{a}$ & $48.2 \pm 0.2$ & $48.3 \pm 0.2$ & $48.4 \pm 0.2$ & 0.771 \\
\hline Chest circumference $(\mathrm{cm})^{\mathrm{a}}$ & $31.6 \pm 0.1$ & $31.5 \pm 0.1$ & $31.9 \pm 0.1$ & 0.098 \\
\hline Head circumference $(\mathrm{cm})^{\mathrm{a}}$ & $33.3 \pm 0.1$ & $33.3 \pm 0.1$ & $33.3 \pm 0.1$ & 0.774 \\
\hline
\end{tabular}

aMean \pm SEM, otherwise $n(\%)$.

'The number of missing data: pre-pregnancy body weight ( 1 and 2 at 23-31 and 35-41 gestational weeks of blood sampling, respectively), pre-pregnancy BMI (1 and 2 at 23-31 and 35-41 gestational weeks of blood sampling, respectively), parity (1 at 32-34 gestational weeks of blood sampling), annual household income (1 at 23-31 and 35-41 gestational weeks of blood sampling, respectively).

Indicates significant difference, $\mathrm{P}<0.05$.

\section{Maternal blood FA status according to different blood sampling periods}

Pronounced differences in absolute FA concentrations in maternal blood were observed throughout pregnancy.
Maternal blood stearic acid content at 35-41 weeks of gestation $(532 \pm 18 \mu \mathrm{g} / \mathrm{mL})$ was lower than that at 32-34 gestational weeks $(629 \pm 24 \mu \mathrm{g} / \mathrm{mL}, \mathrm{P}=0.011$, Figure $1 \mathrm{~B})$. Both palmitoleic and oleic acid levels were significantly higher at $35-41$ gestational weeks $(143 \pm 9 \mu \mathrm{g} / \mathrm{mL}, \mathrm{P}=0.007$ and 

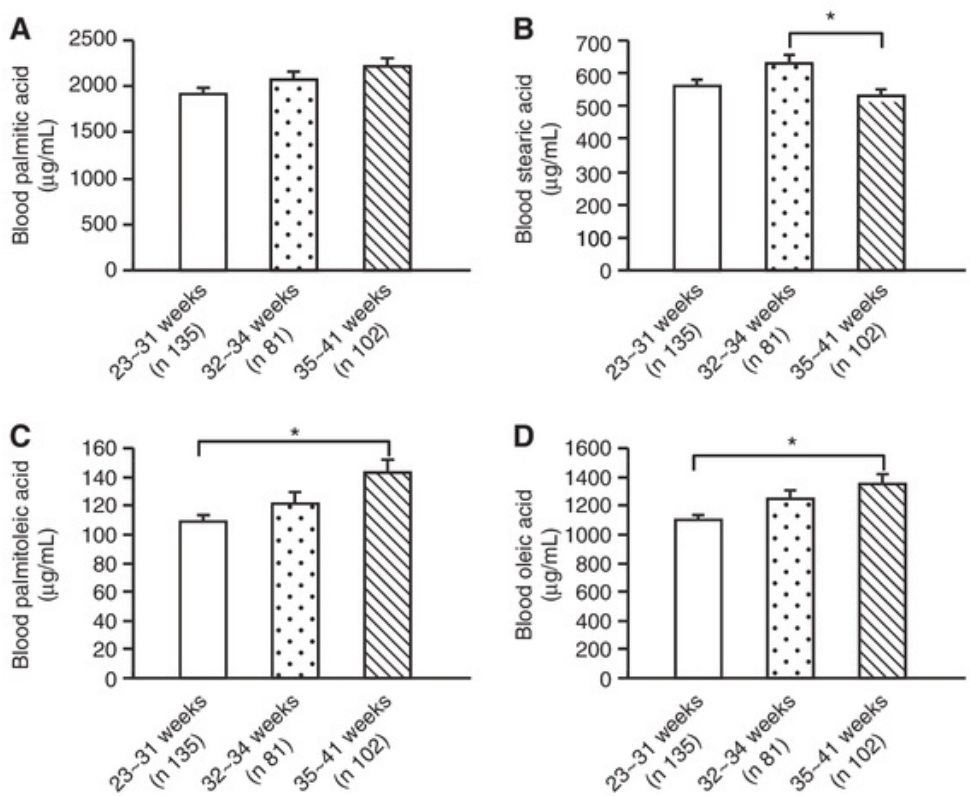

Figure 1 Concentrations of SFAs and MUFAs in maternal blood according to gestational age at blood sampling. Palmitic (A), stearic (B), palmitoleic (C) and oleic (D) acids were measured. ${ }^{\star} P<0.05$. SFAs, saturated fatty acids; MUFAs, monounsaturated fatty acids.

$1350 \pm 70 \mu \mathrm{g} / \mathrm{mL}, \mathrm{P}=0.010$, respectively) compared to those at 23-31 weeks of gestation $(109 \pm 5 \mu \mathrm{g} / \mathrm{mL}$ and $1095 \pm 39$ $\mu \mathrm{g} / \mathrm{mL}$, respectively, Figure $1 \mathrm{C}$ and D). The DHA content at $35-41$ weeks of pregnancy $(25 \pm 2.0 \mu \mathrm{g} / \mathrm{mL})$ was lower than that at 23-31 weeks of gestation $(32 \pm 2.0 \mu \mathrm{g} / \mathrm{mL}, \mathrm{P}=0.029$, Figure 2E). Differences in the EFA status index of mothers were concordant with those observed for DHA content (Figure 2F). We also calculated FA families including SFAs, MUFAs, and n-6/n-3 PUFAs from individual FA (data not shown). The differences observed for total MUFA levels were comparable to those observed for palmitoleic and oleic acids. No significant differences were detected in the total levels of SFAs and of n-6 and n-3 PUFAs.

FA composition ( $\%$ total FAs) in maternal blood during pregnancy is presented in Figures 3 and 4. The proportions of stearic acid in total FAs at 35-41 gestational weeks $(11 \pm 0.3 \%)$ were lower than those at $23-31(13 \pm 0.3 \%$, $\mathrm{P}<0.001)$ and at $32-34(13 \pm 0.4 \%, \mathrm{P}<0.001)$ weeks of gestation (Figure 3B). Interestingly, differences in the proportion of palmitoleic acid were completely opposite to those of stearic acid concentrations in total FAs (Figure 3C). Similar to palmitoleic acid, the proportion of oleic acid was significantly higher at 35-41 weeks of gestation $(26 \pm 0.3 \%)$ compared to the value at $23-31$ weeks of gestation $(24 \pm 0.3 \%, \mathrm{P}<0.001$, Figure $3 \mathrm{D})$. The proportion of AA was lower at $35-41$ weeks of gestation $(1.2 \pm 0.1 \%)$ than at $23-31(1.6 \pm 0.1 \%, \mathrm{P}=0.006)$ and at $32-34(1.6 \pm 0.1 \%$,
$\mathrm{P}=0.049)$ gestational weeks (Figure 4B). The proportion of DHA was lower at $35-41$ weeks of gestation $(0.49 \pm 0.03 \%)$ than at $23-31(0.73 \pm 0.04 \%, P<0.001)$ or $32-34(0.66 \pm 0.05 \%$, $\mathrm{P}=0.021$ ) gestational weeks (Figure $4 \mathrm{E}$ ). We also studied changes in the proportions of FA families (data not shown). The proportion of total MUFAs sharply increased $(28 \pm 0.3 \%, \mathrm{P}=0.001)$ and was similar to the differences observed in oleic acid. The proportion of total n-3 PUFAs was lower at $35-41$ weeks of gestation $(0.91 \pm 0.04 \%)$ than at 23-31 gestational weeks $(1.20 \pm 0.05 \%, \mathrm{P}=0.002)$.

\section{PCA-derived maternal blood FA components}

Considering that total FA concentration was derived from the sum of all measured individual FA, rather than actually determined, the association study used absolute FA contents, not FA proportions, as independent variables. As there were strong correlations among the nine FA contents (Spearman correlation coefficient range: 0.1120.919, data not shown), we performed PCA and identified three components which together represented $83.99 \%$ of the total variation in blood FA concentrations (Table 2). According to the factor loadings of the nine FAs for the three components, strong positive correlations (factor loadings $\geq$ approximately 0.70 ) were observed for Component 1 with palmitic, stearic, palmitoleic and oleic acids, 
A

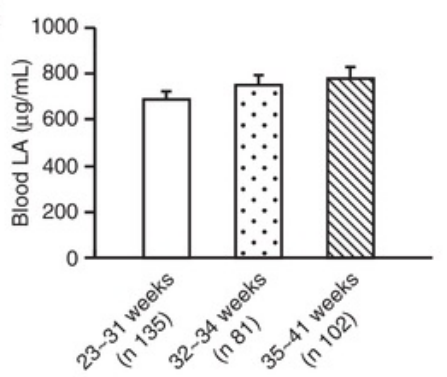

C

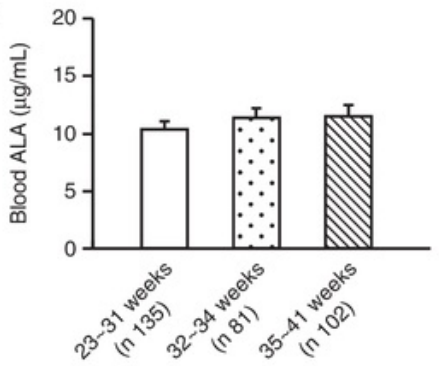

E



B



D
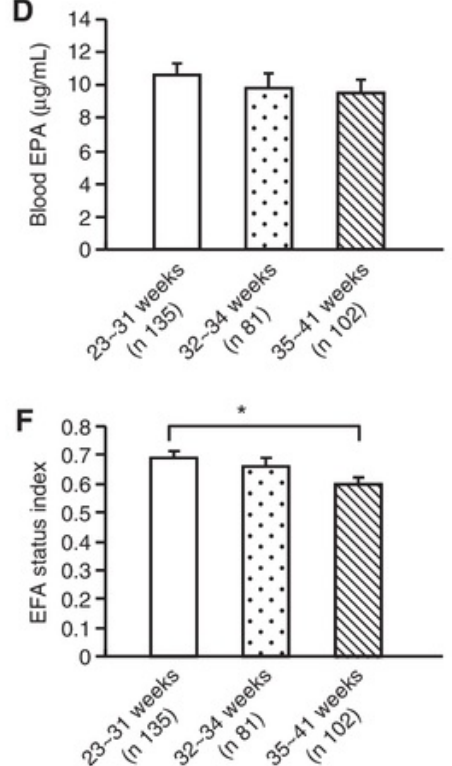

Figure 2 N-6 and n-3 PUFA concentrations in maternal blood according to gestational age at blood sampling. LA (A), AA (B), ALA (C), EPA (D) and DHA (E), and EFA status index (F) were included. *P<0.05. LA, linoleic acid; AA, arachidonic acid; ALA, $\alpha$-linolenic acid; EPA, eicosapentaenoic acid; DHA, docosahexaenoic acid; EFA, essential fatty acid.

for Component 2 with LA, AA, ALA and DHA, and for Component 3 with EPA.

\section{Associations between term birth dimensions and PCA-derived maternal blood FA components}

No significant associations were observed between any birth dimensions and FA components in maternal blood when considering mothers with various blood sampling periods as a whole (data not shown). After stratifying mothers by gestational age at blood sampling, significant associations were detected (Table 3). At 35-41 weeks of gestation, Component 3 , which had strong positive correlation with EPA, was significantly and positively associated with chest circumference in the univariate model $[\beta=0.285 ; 95 \%$ confidence interval $(\mathrm{CI})$ : 0.015, 0.554; $\mathrm{P}=0.039$ ] and in multivariate Model 1 ( $\beta=0.281 ; 95 \% \mathrm{CI}$ : $0.006,0.556 ; \mathrm{P}=0.046)$. The association was marginally significant in multivariate Model 2 ( $\beta=0.264 ; 95 \% \mathrm{CI}$ : $-0.006,0.534 ; \mathrm{P}=0.055)$. No significant associations were found between birth weight, birth length or head circumference and maternal blood FA components at each blood sampling period (all $\mathrm{P}>0.05$, data not shown).

\section{Discussion}

Whole blood lipids are representative of the FA composition of all the circulating lipid classes, lipoproteins and 

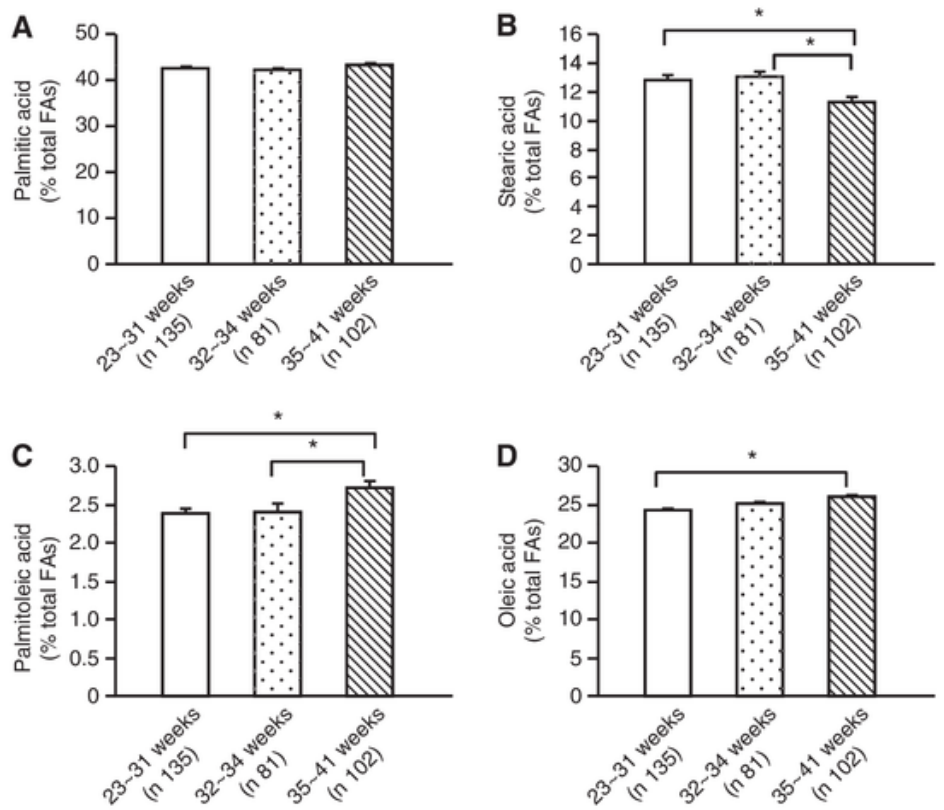

Figure 3 Compositions of SFAs and MUFAs in maternal blood according to gestational age at blood sampling. Palmitic (A), stearic (B), palmitoleic (C) and oleic (D) acids were included. ${ }^{\star} \mathrm{P}<0.05$. SFAs, saturated fatty acids; MUFAs, monounsaturated fatty acids.

cells [19]. Unlike previous studies [3-5], the present study investigated the nine FAs in maternal whole blood at different gestational ages using blood samples from a Japanese pregnancy cohort. Several significant differences in absolute and relative contents of blood FAs were observed during the course of pregnancy. In contrast to stearic acid, levels of palmitoleic and oleic acids were considerably higher at 35-41 gestational weeks relative to 23-31 weeks of gestation. Interestingly, DHA content was lower at 35-41 gestational weeks than at 23-31 weeks of gestation, as was the EFA status index. Taken together, the maternal blood EFA status and DHA level seem to decrease as gestation progresses into the later period of pregnancy, which has also been demonstrated in an earlier longitudinal study [3]. We also explored potential associations between term birth dimensions, i.e., weight, length, and chest and head circumferences, and maternal blood FA status during pregnancy. At 35-41 gestational weeks, FA Component 3 was significantly and positively associated with chest circumference, suggesting a possible novel role of EPA in fetal growth.

Human brain structure, in large part, is composed of lipids (about $50-60 \%$ of dry matter) and includes high proportions of LC-PUFAs, especially DHA and AA [20]. During the last trimester of gestation, a brain growth spurt, accompanied by considerable lipid accretion, occurs in the human fetus [21]. Therefore, it is critical to preferentially transfer LC-PUFAs across the human placenta to support the rapid accretion of LC-PUFAs in nervous tissue during the period of brain growth spurt [4]. Similarly, the present study found that the absolute and relative contents of maternal blood DHA at 35-41 gestational weeks were lowest during pregnancy, as was the proportion of AA. EFA status index also indicated a lower EFA status in maternal blood in this period. Our findings were quite similar to the percentage differences found in total plasma DHA and AA during pregnancy in a cross-sectional study [7], as well as the patterns of maternal plasma phospholipid-associated DHA and AA in longitudinal studies of pregnant women $[3,4]$. We interpreted that these changes in blood FA contents indicated their increased demands in later periods of pregnancy when the nervous system significantly develops.

The differences in relative SFA and MUFA compositions in the course of normal pregnancy have been examined in total plasma, or plasma phospholipids and cholesteryl esters. Relative contents of palmitic acid in maternal total plasma or plasma phospholipids were higher, while those of stearic acid were lower at delivery compared to early and middle pregnancy $[4,7,22]$. Maternal blood stearic acid content and its proportion in our study also were significantly lowered at 35-41 gestational weeks during the course 
A

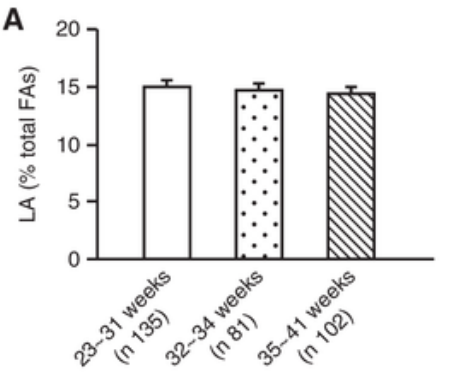

$\mathbf{C}_{0.35}$

要 0.30 - 0.25



E



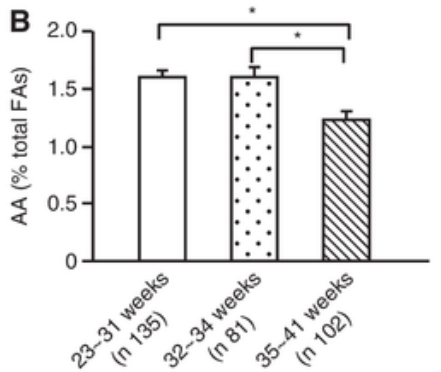

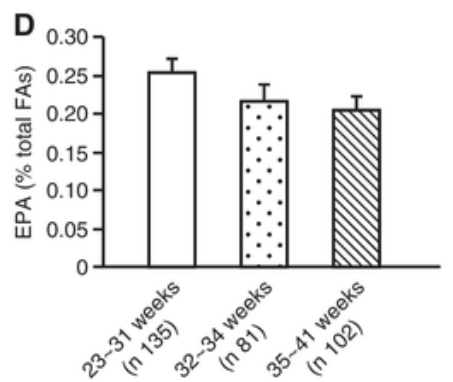

Figure 4 N-6 and n-3 PUFA Compositions in maternal blood according to gestational age at blood sampling. LA (A), AA (B), ALA (C), EPA (D) and DHA (E), were included. *P<0.05. LA, linoleic acid; AA, arachidonic acid; ALA, $\alpha$-linolenic acid; EPA, eicosapentaenoic acid; DHA, docosahexaenoic acid; FAs, fatty acids.

Table 2 Factor loadings of the nine fatty acids (FAs) for the three principal components of FA combinations identified.

\begin{tabular}{|c|c|c|c|}
\hline & Component 1 & Component 2 & Component 3 \\
\hline Variance explained (\%) & 57.15 & 18.35 & 8.49 \\
\hline Eigenvalue & 5.14 & 1.65 & 0.76 \\
\hline Fatty acids & & Factor loadings ${ }^{a}$ & \\
\hline Palmitic acid & 0.95 & 0.25 & 0.02 \\
\hline Stearic acid & 0.70 & 0.27 & 0.22 \\
\hline Palmitoleic acid & 0.88 & 0.10 & 0.03 \\
\hline Oleic acid & 0.91 & 0.26 & -0.02 \\
\hline Linoleic acid & 0.57 & 0.72 & -0.05 \\
\hline Arachidonic acid & 0.21 & 0.89 & 0.20 \\
\hline$\alpha$-linolenic acid & 0.52 & 0.68 & -0.04 \\
\hline EPA & 0.06 & 0.21 & 0.94 \\
\hline $\mathrm{DHA}$ & 0.07 & 0.85 & 0.42 \\
\hline
\end{tabular}

aFactor loading denotes coefficient of the corresponding fatty acid (FA) in the linear combinations of nine FAs for the principal component, and represents the correlation of the component with the corresponding FA.

$E P A=$ eicosapentaenoic acid, $\mathrm{DHA}=$ docosahexaenoic acid. 
Table 3 Associations between fatty acid principal components and chest circumference.

\begin{tabular}{|c|c|c|c|c|}
\hline \multirow{2}{*}{$\begin{array}{l}\text { Gestational age at } \\
\text { blood sampling }\end{array}$} & \multirow{2}{*}{$\begin{array}{l}\text { Independent } \\
\text { variable }\end{array}$} & \multirow[t]{2}{*}{ Univariate model ${ }^{\mathrm{a}}$} & \multicolumn{2}{|r|}{ Multivariate model $^{\mathrm{a}}$} \\
\hline & & & Model $1^{b}$ & Model $2^{c}$ \\
\hline \multirow[t]{3}{*}{ 23-31 weeks } & Component 1 & $0.057(-0.270,0.383)$ & $0.060(-0.240,0.360)$ & $0.068(-0.238,0.373)$ \\
\hline & Component 2 & $-0.057(-0.344,0.229)$ & $-0.048(-0.315,0.218)$ & $-0.089(-0.365,0.187)$ \\
\hline & Component 3 & $0.002(-0.279,0.284)$ & $-0.142(-0.407,0.122)$ & $-0.149(-0.419,0.121)$ \\
\hline \multirow[t]{3}{*}{$32-34$ weeks } & Component 1 & $-0.077(-0.381,0.227)$ & $-0.068(-0.380,0.245)$ & $-0.076(-0.376,0.224)$ \\
\hline & Component 2 & $0.118(-0.128,0.365)$ & $0.106(-0.150,0.362)$ & $0.156(-0.092,0.405)$ \\
\hline & Component 3 & $-0.216(-0.477,0.045)$ & $-0.185(-0.475,0.105)$ & $-0.201(-0.481,0.079)$ \\
\hline \multirow[t]{3}{*}{ 35-41 weeks } & Component 1 & $-0.133(-0.359,0.092)$ & $-0.128(-0.347,0.092)$ & $-0.096(-0.320,0.127)$ \\
\hline & Component 2 & $-0.212(-0.499,0.075)$ & $-0.226(-0.530,0.079)$ & $-0.174(-0.475,0.126)$ \\
\hline & Component 3 & $0.285(0.015,0.554)^{d}$ & $0.281(0.006,0.556)^{d}$ & $0.264(-0.006,0.534)$ \\
\hline
\end{tabular}

'Linear regression analysis with chest circumference as dependent variable and principal component as independent variable. All values are partial regression coefficients $\beta(95 \% \mathrm{Cl})$, representing the expected change in chest circumference as a result of a unit change in component score.

bAdjusted for maternal age, height, weight before pregnancy, parity, gestational age at birth, gestational age at blood sampling, and infant gender.

'As in Model 1 with additional adjustment for smoking and alcohol intake during pregnancy, and annual household income.

Indicates significant associations, $\mathrm{P}<0.05$.

of pregnancy. Unlike aforementioned studies [4, 7, 22], no significant increment was observed in absolute and relative contents of palmitic acid during pregnancy. Additionally, the proportions of plasma cholesteryl ester-associated and total plasma oleic acid increased as gestation progressed $[4,7]$. In the present study, absolute and relative amounts of blood oleic and palmitoleic acids were higher at 35-41 gestational weeks compared to 23-31 and/or 32-34 weeks of gestation. Thus, a loss of LC-PUFAs such as DHA and AA in maternal blood at 35-41 weeks of gestation might have been replaced by MUFAs rather than SFAs [4, 22].

The observed differences in maternal blood FA status during pregnancy could be related to changes in dietary intake of FAs. However, this would be unlikely as others reported that dietary habits remained unaltered during pregnancy. Neither the amount and type of fat nor the FA composition of the maternal diet changed during pregnancy until 1 month postpartum [23, 24]. Other previous studies indicated changes in estrogen levels and intrahepatic cholestasis during normal pregnancy possibly explained the altered patterns of palmitic, stearic, and oleic acids, LA and AA in maternal plasma phospholipids during pregnancy $[4,5]$. This speculation remains to be clarified as we did not measure estrogen levels or markers for cholestasis.

The associations of maternal plasma EFAs and their LC-PUFA derivatives in the course of pregnancy with anthropometric parameters of newborns have been extensively investigated, especially for DHA and AA in terms of birth weight, birth length and head circumference [3, 9-11, 25]. It would be worth emphasizing that our study examined nine FAs representative for SFAs, MUFAs and PUFAs simultaneously in relation to the anthropometric parameters. Namely, in an attempt to control potential confounding of metabolic interactions among these nine FAs, multivariate association analysis using principal components derived from the nine FAs was performed. One novel finding that a unit increase in the Component 3 at 35-41 gestational weeks was associated with an increase in chest circumference might indicate potential influence of falling EPA in maternal blood as gestation processes on fetal chest circumference. The finding may be consistent with an earlier studies that low maternal plasma phospholipid-associated EPA during early pregnancy was associated with lower birth weight $[9,25]$. However, caveat is needed to interpret the present finding as there could be a possibility of chance finding as a result of multiple tests done in the present study, although the comparisons had been determined before conducting the study. Also, we did not solely rely on the statistical significance of the findings, but on biological plausibility and consistency.

Meanwhile, it is not clear why only associations with chest circumference, without other birth dimensions, were observed in the present study. One speculation may be our use of PCA-derived components in the multivariate analysis instead of absolute FA levels. Another reason may be related to the fact that the present study was carried out in Japanese whose fish consumption is generally much higher than other ethnicities. Indeed, a previous study indicated that blood n-3 PUFA level was much higher in Korean people - who eat a lot of marine products, as do Japanese people - than in Americans [26]. There may be a threshold in the association between $n-3$ 
PUFA and anthropometric parameters. Finally, statistical power might have been insufficient to detect small effect so larger studies would be needed in future.

There are some limitations in this study. First, although this is a part of the established Hokkaido Cohort Study, the analyses of maternal blood FA status in the course of gestation are cross-sectional in nature. Second, this study has a small sample size that may limit its statistical efficiency. Third, selection bias may have occurred because this cohort was based in a single hospital that treated pregnant women in Sapporo and the surrounding areas. Fourth, this study lacks dietary assessment of pregnant women.

\section{Conclusions}

In conclusion, several significant differences in blood FA status were observed during normal pregnancy. In particular, the absolute and/or relative contents of DHA and AA in maternal blood were lower at 35-41 weeks of gestation, while those of MUFAs were higher. Moreover, the blood FA Component 3 representing a strong positive correlation with EPA was significantly and positively associated with chest circumference at 35-41 gestational weeks after adjustment for confounders. These results may well suggest that PUFA intake during pregnancy should be increased to meet the fetal requirement for growth and development, and that maternal blood EPA content may be involved in fetal growth.

Authors' contributions: XJ carried out the analysis, interpreted the results, and drafted the manuscript. HY carried out the analysis. MT, HN, YH, and HY participated in the measurement of maternal blood fatty acid levels. SS, AA, CM, TI, and RK participated in the design of the Hokkaido Cohort Study and carried it out. TN conceived the study, interpreted the results, and contributed to drafting the manuscript. All authors read and approved the final manuscript.

Acknowledgments: This work was funded by Grants-inAid for Health Scientific Research from the Japan Society for the Promotion of Science (25253050). Additionally, we would like to thank the medical staff and the participants at Sapporo Toho Hospital, and staff of the "Hokkaido Study on Environment and Children's Health". We also thank Ms Aiko Tajima at the Department of Occupational and Environmental Health of Nagoya University Graduate School of Medicine for the maternal blood TG and FA measurements.

\section{References}

[1] Guillou H, Zadravec D, Martin PG, Jacobsson A. The key roles of elongases and desaturases in mammalian fatty acid metabolism: insights from transgenic mice. Prog Lipid Res. 2010;49:186-99.

[2] Ortega-Senovilla H, Alvino G, Taricco E, Cetin I, Herrera E. Enhanced circulating retinol and non-esterified fatty acids in pregnancies complicated with intrauterine growth restriction. Clin Sci (Lond). 2009;118:351-8.

[3] Al MD, van Houwelingen AC, Kester AD, Hasaart TH, de Jong AE, Hornstra G. Maternal essential fatty acid patterns during normal pregnancy and their relationships to the neonatal essential fatty acid status. Br J Nutr. 1995;74:55-68.

[4] De Vriese SR, Dhont M, Christophe AB. FA composition of cholesteryl esters and phospholipids in maternal plasma during pregnancy and at delivery and in cord plasma at birth. Lipids. 2003;38:1-7.

[5] De Vriese SR, Christophe AB, Maes M. Fatty acid composition of phospholipids and cholesteryl esters in maternal serum in the early puerperium. Prostaglandins Leukot Essent Fatty Acids. 2003;68:331-5.

[6] Matorras R, Ruiz JI, Perteagudo L, Barbazan MJ, Diaz A, Valladolid A, et al. Longitudinal study of fatty acids in plasma and erythrocyte phospholipids during pregnancy. J Perinat Med. 2001;29:293-7.

[7] Sanjurjo P, Matorras R, Ingunza N, Alonso M, RodriguezAlarcon J, Perteagudo L. Cross-sectional study of percentual changes in total plasmatic fatty acids during pregnancy. Horm Metab Res. 1993;25:590-2.

[8] Godfrey KM, Barker DJ. Fetal nutrition and adult disease. Am J Clin Nutr. 2000;71(5 Suppl):1344S-52S.

[9] van Eijsden M, Hornstra G, van der Wal MF, Vrijkotte TG, Bonsel GJ. Maternal n-3, n-6, and trans fatty acid profile early in pregnancy and term birth weight: a prospective cohort study. Am J Clin Nutr. 2008;87:887-95.

[10] Dirix CE, Kester AD, Hornstra G. Associations between neonatal birth dimensions and maternal essential and trans fatty acid contents during pregnancy and at delivery. $\mathrm{Br}$ J Nutr. 2009;101:399-407.

[11] Rump P, Mensink RP, Kester AD, Hornstra G. Essential fatty acid composition of plasma phospholipids and birth weight: a study in term neonates. Am J Clin Nutr. 2001;73:797-806.

[12] Kishi R, Sasaki S, Yoshioka E, Yuasa M, Sata F, Saijo Y, et al. Cohort profile: the Hokkaido study on environment and children's health in Japan. Int J Epidemiol. 2011;40:611-8.

[13] Konishi K, Sasaki S, Kato S, Ban S, Washino N, Kajiwara J, et al. Prenatal exposure to PCDDs/PCDFs and dioxin-like PCBs in relation to birth weight. Environ Res. 2009;109:906-13.

[14] Washino N, Saijo Y, Sasaki S, Kato S, Ban S, Konishi K, et al. Correlations between prenatal exposure to perfluorinated chemicals and rediced fetal growth. Environ Health Perspect. 2009;117:660-7.

[15] Nakashima R, Hayashi Y, Md K, Jia X, Wang D, Naito H, et al. Exposure to DEHP decreased four fatty acid levels in plasma of prepartum mice. Toxicology. 2013;309:52-60.

[16] Folch J, Lees M, Sloane Stanley GH. A simple method for the isolation and purification of total lipids from animal tissues. J Biol Chem. 1957;226:497-509. 
[17] Anderson SG, Sanders TA, Cruickshank JK. Plasma fatty acid composition as a predictor of arterial stiffness and mortality. Hypertension. 2009;53:839-45.

[18] Wheeler SJ, Poston L, Thomas JE, Seed PT, Baker PN, Sanders TA. Maternal plasma fatty acid composition and preg. nancy outcome in adolescents. Br J Nutr. 2011;105:601-10.

[19] Agostoni C, Galli C, Riva E, Rise P, Colombo C, Giovannini M, et al. Whole blood fatty acid composition at birth: from the maternal compartment to the infant. Clin Nutr. 2011;30:503-5.

[20] Lauritzen L, Hansen HS, Jorgensen MH, Michaelsen KF. The essentiality of long chain n-3 fatty acids in relation to development and function of the brain and retina. Prog Lipid Res. 2001;40:1-94.

[21] Larque E, Demmelmair H, Gil-Sanchez A, Prieto-Sanchez MT, Blanco JE, Pagan A, et al. Placental transfer of fatty acids and fetal implications. Am J Clin Nutr. 2011;94(6 Suppl):1908S-13S.

[22] De Vriese SR, Houwelingen AC, Hornstra G, Dhont M, Christophe AB. The composition of saturated fatty acids in plasma phospholipids changes in a way to counteract changes in the mean melting point during pregnancy. Lipids. 2001;36:15-20.
[23] Al MD, Badart-Smook A, von Houwelingen AC, Hasaart TH, Hornstra G. Fat intake of women during normal pregnancy: relationship with maternal and neonatal essential fatty acid status. J Am Coll Nutr. 1996;15:49-55.

[24] De Vriese SR, De Henauw S, De Backer G, Dhont M, Christophe AB. Estimation of dietary fat intake of Belgian pregnant women: comparison of two methods. Ann Nutr Metab. 2001;45:273-8.

[25] Smits LJ, Elzenga HM, Gemke RJ, Hornstra G, van Eijsden M. The association between interpregnancy interval and birth weight: what is the role of maternal polyunsaturated fatty acid status? BMC Pregnancy Childbirth. 2013;13:23.

[26] Sekikawa A, Shin C, Masaki KH, Barinas-Mitchell EJ, Hirooka N, Willcox BJ, et al. Association of total marine fatty acids, eicosapentaenoic and docosahexaenoic acids, with aortic stiffness in Koreans, Whites, and Japanese Americans. Am J Hypertens. 2013;26:1321-27.

The authors stated that there are no conflicts of interest regarding the publication of this article. 
Association of maternal whole blood fatty acid status during the prenatal period with term birth dimensions: a cross-sectional study

ORIGINALITY REPORT

0

$0 \%$

INTERNET SOURCES
$6 \%$

PUBLICATIONS
$0 \%$

STUDENT PAPERS
Exclude quotes

On

Exclude bibliography

On
Exclude matches

$<3 \%$ 